\title{
Comparative Analysis of Pediatric and Adult Leptospirosis in Pleven Region, Bulgaria
}

\author{
Galya Ivanova Gancheva \\ Department of Infectious Diseases, Epidemiology, Parasitology and Tropical Medicine, Faculty of Public Health, Medical University, \\ Pleven, Bulgaria
}

Email address:

galya_gancheva@abv.bg

\section{To cite this article:}

Galya Ivanova Gancheva. Comparative Analysis of Pediatric and Adult Leptospirosis in Pleven Region, Bulgaria. Science Journal of Clinical Medicine. Vol. 6, No. 5, 2017, pp. 91-97. doi: 10.11648/j.sjcm.20170605.16

Received: February 18, 2017; Accepted: March 11, 2017; Published: October 24, 2017

\begin{abstract}
Leptospirosis is worldwide re-emerging zoonosis with protean clinical manifestations. The more common mild, anicteric form is characterized by nonspecific symptoms such as fever, headache, chills, myalgia, nausea and abdominal pain. The severe, potentially fatal form (Weil's disease) typically presenting as triad of jaundice, acute renal failure (ARF), and hemorrhage is estimated to occur in $5-15 \%$ of all human infections with mortality rate up to $40 \%$. Elder age is one of poor prognostic factors in leptospirosis. There are few studies about leptospirosis in children and no one comparative study of pediatric and adult leptospirosis in regions with temperate climate. Our aims were to describe retrospectively the disease spectrum and outcome differences in children and adults with leptospirosis over a period of 41 years and to compare severity in different age groups. The study material comprised the data from 105 consecutive leptospirosis cases from Central Northern Bulgaria treated in Clinic of Infectious Diseases at University Hospital - Pleven (1976-2016) (95 male, age 37 18 years, lethal outcome in $12.38 \%$ ), followed by comparative analysis of group of children (age $\leq 17$-years-old; $\mathrm{N}_{1}=13$ ) versus group of adults (age $\geq 18$ years-old; $\left.\mathrm{N}_{2}=92\right)$. Results: Fever $(100 \%)$, hepatomegaly $(92 \%)$, conjunctival suffusions $(88 \%)$, myalgia $(87 \%)$, nausea and vomiting $(84 \%)$, shivering $(79 \%)$, splenomegaly $(74 \%)$, headache $(65 \%)$, jaundice $(64 \%)$, oliguria $(58 \%)$, tachycardia (55\%), hypotension (50\%), abdominal pain (41\%), and hemorrhagic diathesis (37\%) were the most characteristic manifestations in whole series. Only headache and meningitis had had a significantly higher prevalence in the group of children. Leucocytosis, blood urea nitrogen, serum creatinine, serum bilirubin, aminotransferases, gamma glutamyl tranferase (GGT) and serum amylase levels were significantly higher in group of adults. Potassium level, total protein and albumins were significantly lower in the same group. The distribution of cases in whole series according to severity was as follows: mild cases -27 , moderate -44 cases and severe -34 cases. In group of children seven mild and six moderate cases had been observed versus 20 mild, 38 moderate and 34 severe cases in the group of adults. All thirteen deceased patients were adults. We concluded that in Pleven region, leptospirosis in children usually is mild and anicteric. The leptospiral meningitis is not unusual manifestation for pediatric leptospirosis. The major factors leading to death in adults with leptospirosis were lung edema and brain edema due to severe ARF.
\end{abstract}

Keywords: Leptospirosis, Children, Adults, Renal Involvement, Hepatic Dysfunction, Meningitis

\section{Introduction}

Leptospirosis, a worldwide zoonosis is caused by pathogenic leptospires, belonging to Leptospira interrogans complex. Humans are accidental hosts and usually become infected through contact with water or soil contaminated by the urine of infected animals such as rodents, dogs, cattle and pigs. Exposure of skin or mucous membranes to leptospires can lead to infection. The clinical manifestations of leptospirosis are protean and range from subclinical to potentially fatal disease. After incubation period of 2 to 20 days leptospirosis manifests as biphasic illness consisting of initial leptospiremic phase (lasting 3 to 7 days) followed by immune phase (4 to 30 days). The more common mild, anicteric form is characterized by nonspecific symptoms such as fever, headache, chills, myalgia, nausea and abdominal pain. The severe, potentially fatal form (Weil's disease) is 
estimated to occur in $5-15 \%$ of all human infections, typically presenting as triad of jaundice, acute renal failure (ARF), and hemorrhage [1]. A recent systematic review estimated that there are 1.03 (95\% CI $0.43-1.75)$ million cases of leptospirosis worldwide each year and 58,900 deaths $(95 \%$ CI 23,800-95,900) [2, 3], which corresponds to an estimated 2.9 million disability-adjusted life years per annum, including 2.8 million years of life lost due to premature death [4].

Worldwide, independent prognostic factors for lethal outcome in leptospirosis have been found to include older age, oliguria, hyperkalemia, abnormal serum creatinine, acute respiratory distress syndrome (ARDS), pulmonary hemorrhage, elevated bilirubin, hypotension, arrhythmia, and altered mental status [5-9]. However, such studies have typically been hospital-, not population-based. Intrinsic virulence variations among serovars have been claimed to partially explain disease severity albeit mild and severe forms may be caused by a broad range of pathogenic serovars [10]. Delay between onset and hospitalization have also been highlighted as determinants of poor outcome.

In Bulgaria, leptospirosis is a reportable disease since 1952, when a database and official registration was initiated. A mean annual incidence rate of 0.9 to 3.1 per 100000 was reported during the period 1953-1968 followed by decreasing to 0.1 per 100000 population within next ten years. Since 1976, a mean annual incidence of 0.37 per 100000 was reported [11], decreasing to 0.18/100 000 (2006-2009) [12]. Because the clinical presentation of leptospirosis varies in different geographic areas, and the fatality rate in severe course is significant, a better understanding of clinical presentation of leptospirosis is needed to enhance its recognition and appropriate treatment. Predictors of lethal outcome must be evaluated in each clinico-epidemiologic setting to consider regional peculiarities [13]. Contrasting to considerable number of studies upon leptospirosis in adults, there are few studies about leptospirosis in children, especially in regions with temperate climate.

Our objectives were to describe disease spectrum and outcome differences in children and adults admitted for leptospirosis and to compare severity in different age groups.

\section{Materials and Methods}

We performed retrospective study of all consecutive leptospirosis cases treated after written informed consent in Clinic of Infectious Diseases at University Hospital - Pleven (1976-2015) $(\mathrm{n}=105$, lethal outcome in $13 / 105 ; 12.38 \%)$. A retrospective database for patients presenting with leptospirosis (1976-1984) was initiated and continued prospectively to the December $31^{\text {st }}$ 2016. Subjects were screened by microscopic agglutination test (MAT) for leptospirosis (in the National Reference Laboratory at National Center of Infectious and Parasitic Diseases - Sofia). A positive diagnosis was confirmed if an initial titer of $\geq 100$ for MAT was observed.

The data was obtained through the medical documentation of cases and the protocol included review of medical records, with description of demographic, epidemiologic, clinical, and laboratory data. The cases were grouped in two age groups children (age $\leq 17$ years), and adults (age $\geq 18$ years) [14]. Comparative analysis of clinical manifestations, laboratory parameters and outcome in both age groups was performed. The data were analyzed using the Statgraphics Plus Version 2. 1. package. We used the t-test and for non-parametric distributions, the $\chi^{2}$ test; $\mathrm{p}<0.05$ was considered to be significant.

Severity of cases was complexly assessed as mild, moderate and severe according to the following definitions [11]: Mild form of leptospirosis had been defined at mild to moderate intoxication, anicteric or mild icteric, without hemorrhagic diathesis, without involvement of respiratory, cardiac and central nervous system (CNS), with mild renal dysfunction without acute renal failure (ARF).

Moderate form of leptospirosis had been defined at markedly demonstrated intoxication, moderate jaundice, skin hemorrhages, transitory cardiovascular abnormalities without myocardial dysfunction, ARF improving without dialysis.

Severe leptospirosis had been defined at severe intoxication, intensive jaundice with severe hepatic dysfunction, skin hemorrhages and visceral bleeding, toxic myocarditis, severe ARF requiring dialysis, respiratory dysfunction and CNS-involvement.

We used $\varphi$-coefficient by modified Pearson's test about correlation of severity with age (interpreted by three-grade score as follows: weak correlation at $\varphi<0.3$, moderate $0.31<\varphi<0.7$ and strong $-\varphi \geq 0.7$ ). Odds ratios (ORs) had been calculated.

This study was approved by the Ethics Committee of Medical University - Pleven. We examined only stored data of participants from mentioned above database. Written informed consent was obtained from all cases.

\section{Results}

One hundred and five patients - mean age $37 \pm 18(8-78)$ years, 95 males $(90.48 \%$ ), and urban residents $62.86 \%$, with leptospirosis were treated in Clinic of Infectious Diseases at University Hospital - Pleven, Bulgaria since January $1^{\text {st }} 1976$ to December $31^{\text {st }}$ 2016. The mean annual incidence of leptospirosis for 36-year period in Pleven' region was 0.37 per $100 \quad 000$ population (Figure 1). Summer seasonal predominance (83 cases; 79.04\%) was observed - number of cases during June, July, August and September was 12, 30, 29 and 12 , respectively.

The age distribution of cases was as follows: children age $\leq 17$ years old $\left(\mathrm{n}_{1}=13 ; 12.38 \%\right.$ of all cases $)$ and adults age $\geq 45$ years $\left(n_{2}=92 ; 87.62 \%\right.$ of all cases $)$.

The gender distribution of cases was as follows: in children group male-to-female ratio was 5.5 to 1.0 (11 to 2 cases) and in group of adults this ratio was 10.5 to 1.0 (84 to 8 cases, respectively) $(\mathrm{p}>0.05)$.

Epidemiological analysis revealed that 93 (88.57\%) of all cases had exposures to risk factors for leptospirosis. All of 
children and 80 of adults $(86.96 \%)$ had risk exposures to one or more than one risk factors in different recreational (fishing, swimming, bathing) or professional (handling or pasturing of livestock, cleaning of sewages, cutting of grasses, slaughtering etc.) activities.

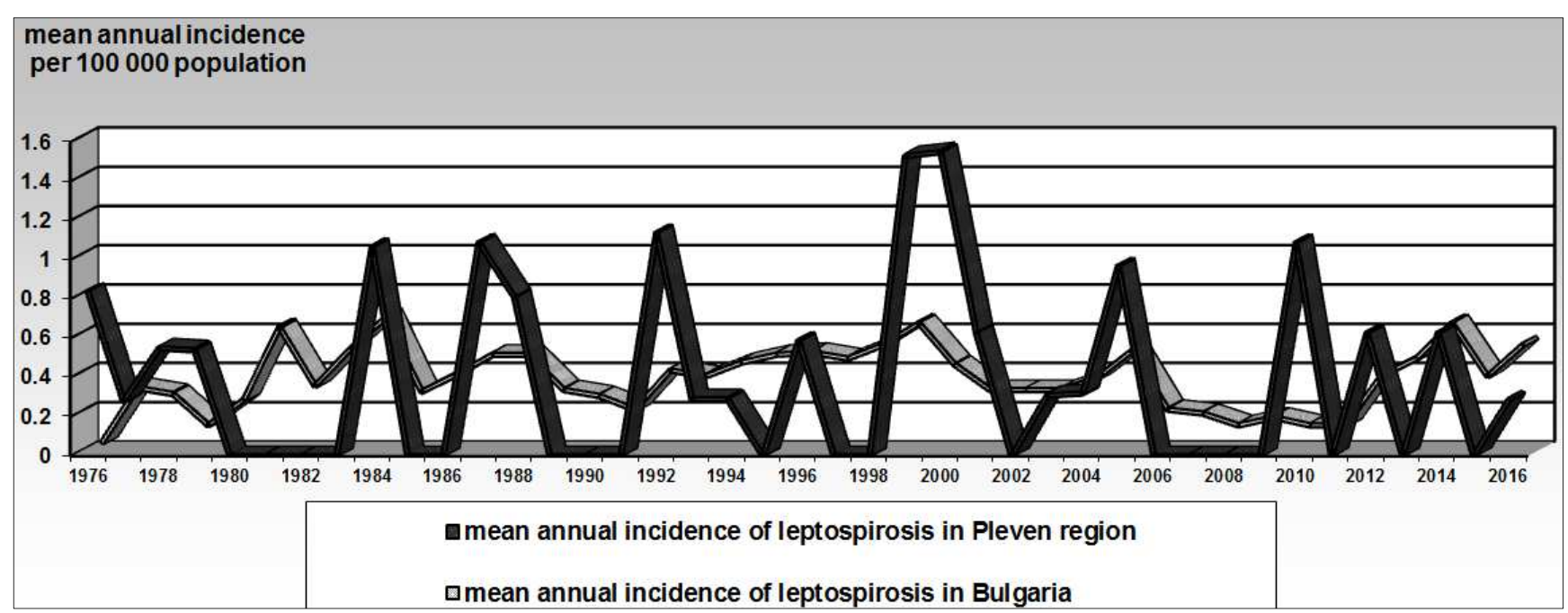

Figure 1. Mean Annual Incidence of leptospirosis in Bulgaria and Pleven region (1976-2011).

The characteristic of risk exposures in whole series and separately in different age groups are shown on Table 1.

Table 1. Leptospirosis in Pleven region (1976-2016) - risk exposures in whole series and compared in children and adults.

\begin{tabular}{|c|c|c|c|c|}
\hline Features & Whole series n (\%) & Children $\mathrm{n}_{1}(\%)$ & Adults $n_{2}(\%)$ & $\bar{p}$ \\
\hline $\begin{array}{l}\text { Presence of risk exposure } \\
\text { Risk factors }\end{array}$ & $93(88.57)$ & $13(100)$ & $80(86.96)$ & $<0.0005$ \\
\hline Contaminated water & $64(60.95)$ & $12(92.31)$ & $52(56.52)$ & $<0.0005$ \\
\hline Rodents & $25(23.81)$ & - & $25(27.17)$ & $<0.0005$ \\
\hline $\begin{array}{l}\text { Livestock } \\
\text { Exposure to risk factors } \mathrm{p}\end{array}$ & $16(15.24)$ & $1(7.69)$ & $15(16.30)$ & n.s. \\
\hline One factor & $80(76.19)$ & $13(100)$ & $67(72.83)$ & $<0.0005$ \\
\hline Recreational & $52(49.52)$ & $12(92.31)$ & $40(43.48)$ & $<0.0005$ \\
\hline Occupational & $44(41.90)$ & $1(7.69)$ & $43(46.74)$ & $<0.0005$ \\
\hline
\end{tabular}

$\mathrm{n}$ - number of cases; n.s. - non significant.

Fever $(100 \%)$, hepatomegaly $(92.38 \%)$, conjunctival suffusions $(87.62 \%)$, myalgia $(86.67 \%)$, nausea and vomiting $(83.81 \%)$, shivering (79.05\%), splenomegaly $(74.29 \%)$, headache $(64.76 \%)$, jaundice $(63.81 \%)$, oliguria $(58.10 \%)$, tachycardia (55.24\%), hypotension (49.52\%), abdominal pain (40.95\%), and hemorrhagic diathesis $(37.14 \%)$ were the most characteristic manifestations in whole series. Only headache had had a significantly higher prevalence in the group of children. Jaundice, oligo/anuria, hemorrhagic diathesis, abdominal pain and splenomegaly had been registered more frequently in group of adults. Diarrhea was reported only in adults. Acute renal failure had registered in two of children (15.38\%) versus $67.39 \%$ of adults. Involvement of CNS as meningitis was found in four of children (30.77\%) vs. 17 of adults (18.48\%). Myocarditis, acute respiratory failure and pancreatitis was observed only in group of adults $(22.83 \%$, $15.22 \%$ and $7.61 \%$, respectively). The prevalence of symptoms and syndromes in whole series and separately in the age groups is shown on Table 2.

The comparative analysis of laboratory findings in different age groups revealed that number of leucocytes and levels of blood urea nitrogen, serum creatinine, serum bilirubin, aminotransferases, gamma glutamyl tranferase (GGT) and serum amylase were significantly higher in group of adults. Potassium level, total protein and albumins were significantly lower in the same group. These results demonstrate that renal and hepatic functions are affected manifestly in adults (Table 3 ).

According to the definitions for mild, moderate and severe leptospirosis mentioned above and after assessment of clinical and laboratory findings showed above, we established the following distribution of cases in whole series: 27 mild cases, 44 moderate cases and 34 severe cases. In group of children seven mild and six moderate cases had been observed versus 20 mild, 38 moderate and 34 severe cases in the group of adults. Distribution of leptospirosis cases according to severity compared in group of children and adults revealed that mild leptospirosis is priority of children group whereas severe leptospirosis is characteristic for adults $(\mathrm{p}<0.05)$ (Fig. 2). 
We used $\varphi$-coefficient by modified Pearson's test (interpreted by three-grade score as follows: weak correlation at $\varphi<0.3$, moderate $0.31<\varphi<0.7$ and strong $-\varphi \geq 0.7$ ) about correlation of severity with age. Mild correlation of severity with children age was established $(\varphi=0.53)$ and strong - in group of adults $(\varphi=0.84)$.

Table 2. Leptospirosis in Pleven region (1976-2016) - prevalence of symptoms and syndromes in whole series and compared in children and adults.

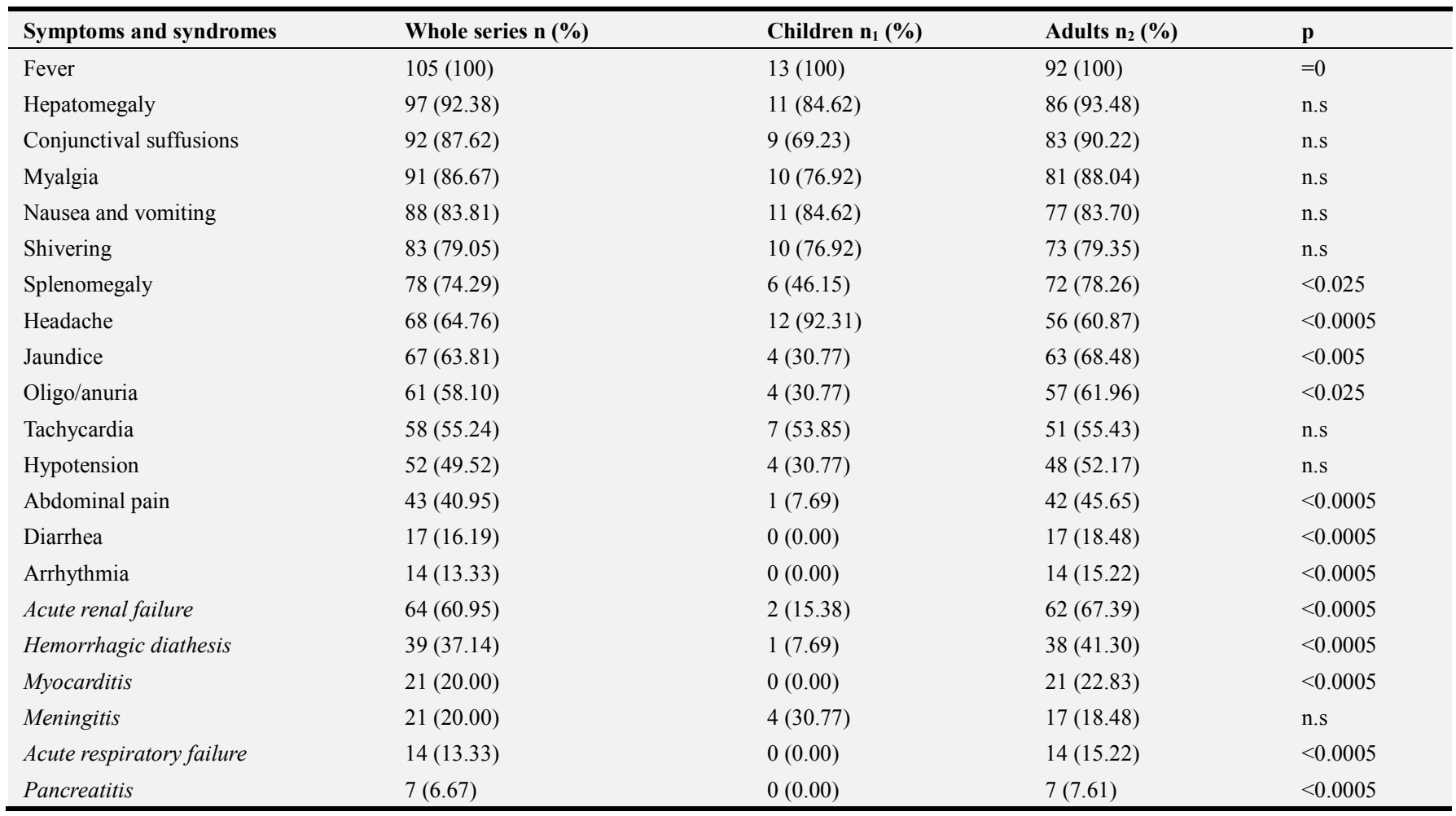

$\mathrm{n}$ - number of cases; n.s. - non significant.

Table 3. Leptospirosis in Pleven region (1976-2016) - laboratory findings in whole series and compared in children and adults.

\begin{tabular}{|c|c|c|c|c|c|}
\hline Parameter & $\begin{array}{l}\text { Reference } \\
\text { value }\end{array}$ & $\begin{array}{l}\text { Whole series - mean } \pm \text { SD } \\
(\text { min-max) }\end{array}$ & $\begin{array}{l}\text { Children - mean } \pm \text { SD } \\
(\text { min-max })\end{array}$ & $\begin{array}{l}\text { Adults - mean } \pm \text { SD } \\
(\text { min-max })\end{array}$ & $\mathbf{p}$ \\
\hline Hemoglobin $(\mathrm{g} / \mathrm{L})$ & $120-188$ & $131 \pm 19(65-168)$ & $130 \pm 10.4(114-147)$ & $131 \pm 20(65-168)$ & n.s. \\
\hline Leucocytes (cells x 109/L) & $4.0-10.0$ & $13.6 \pm 6.5(2.9-32)$ & $9.0 \pm 4.2(2.9-15.3)$ & $14.2 \pm 6.5(2.9-32)$ & $<0.0005$ \\
\hline Platelets (cells x 109/L) & $150-400$ & $151 \pm 115(8-594)$ & $150 \pm 84(17-256)$ & $151 \pm 118(8-594)$ & n.s. \\
\hline Urea (mmol/L) & $1.7-8.3$ & $22.4 \pm 16.5(2.8-98.6)$ & $11.0 \pm 3.4(7.8-17.8)$ & $23.6 \pm 16.9(2.8-98.6)$ & $<0.0005$ \\
\hline Creatinine $(\mu \mathrm{mol} / \mathrm{L})$ & $44.2-134$ & $290.4 \pm 201.2(56-818)$ & $141.0 \pm 67(88-274)$ & $305.6 \pm 204.2(56-818)$ & $<0.0005$ \\
\hline $\mathrm{K}^{+}(\mathrm{mmol} / \mathrm{L})$ & $3.5-5.6$ & $4.1 \pm 0.8(2.6-6.5)$ & $4.3 \pm 0.4(3.7-4.9)$ & $4.1 \pm 0.8(2.6-6.5)$ & $<0.0005$ \\
\hline $\mathrm{Na}^{+}(\mathrm{mmol} / \mathrm{L})$ & $130-151$ & $137.3 \pm 7.2(112-155)$ & $137.9 \pm 6.3(126-146)$ & $137.2 \pm 7.3(112-155)$ & n.s. \\
\hline Total bilirubin $(\mu \mathrm{mol} / \mathrm{L})$ & $3.4-21$ & $154.2 \pm 148.3(3.1-801)$ & $51.1 \pm 38(7.2-129)$ & $167.2 \pm 173.8(3.1-801)$ & $<0.0005$ \\
\hline Direct bilirubin $(\mu \mathrm{mol} / \mathrm{L})$ & $0.8-8.5$ & $134.8 \pm 129.4(2.5-564)$ & $35.2 \pm 24.3(7.2-83)$ & $146.7 \pm 131.8(2.5-564)$ & $<0.0005$ \\
\hline ASAT (IU/L) & $\leq 37$ & $108.6 \pm 106.3(6-625)$ & $36.5 \pm 17.3(12-69)$ & $119.3 \pm 110.9(6-625)$ & $<0.0005$ \\
\hline ALAT (IU/L) & $\leq 40$ & $95.1 \pm 77.1(11-382)$ & $33.7 \pm 27.5(12-111)$ & $103.5 \pm 78(11-382)$ & $<0.0005$ \\
\hline GGT (IU/L) & $15-28$ & $182.6 \pm 169.0(16-637)$ & $72.2 \pm 62.8(16-196)$ & $191.7 \pm 171.7(27-637)$ & $<0.025$ \\
\hline Alkaline phosphatase (IU/L) & $50-260$ & $302.6 \pm 234.0(37-1431)$ & $211 \pm 128.8(150-436)$ & $307.3 \pm 243.2(37-1431)$ & n.s. \\
\hline Lactatedehydrogenase (IU/L) & $100-360$ & $918.4 \pm 550.5(287-2305)$ & - & $918.4 \pm 550.5(287-2305)$ & NA \\
\hline Creatine kinase (IU/L) & $80-190$ & $1980.2 \pm 1265.2(9-10438)$ & - & $1980.2 \pm 1265.2(9-10438)$ & NA \\
\hline Total protein $(\mathrm{g} / \mathrm{L})$ & $58-80$ & $64.0 \pm 9.55(38.1-87)$ & $71.9 \pm 6.1(64-80.5)$ & $63.2 \pm 9.5(38.1-87)$ & $<0.001$ \\
\hline Albumins (g/L) & $35-55$ & $35.5 \pm 8.0(18.5-51)$ & $42.8 \pm 7.3(33.5-51)$ & $35.1 \pm 7.9(18.5-51)$ & $<0.025$ \\
\hline C-reactive protein $(\mathrm{mg} / \mathrm{dL})$ & $0-5$ & $208.3 \pm 199.8(29.5-459)$ & - & $208.3 \pm 199.8(29.5-459)$ & NA \\
\hline Fibrinogen $(\mathrm{g} / \mathrm{L})$ & $2.0-4.5$ & $6.8 \pm 2.4(1.4-12)$ & $6.8 \pm 2.4(3.9-11.9)$ & $6.8 \pm 2.4(1.4-12)$ & n.s. \\
\hline Prothrombin index $(\%)$ & $80-110$ & $86 \pm 17(24-114)$ & $81 \pm 14(61-93)$ & $87 \pm 18(24-114)$ & n.s. \\
\hline Serum amylase (IU/L) & $30-300$ & $434 \pm 394(28.8-2302)$ & $114.5 \pm 24.7(97-132)$ & $445 \pm 398(28.8-2302)$ & $<0.0005$ \\
\hline
\end{tabular}

$\mathrm{n}$ - number of cases; n.s. - non significant; NA - not available. 


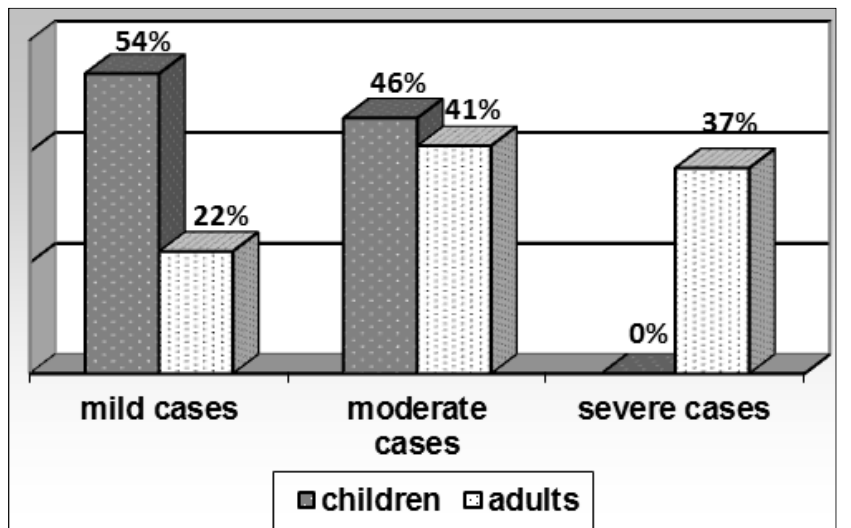

Figure 2. Distribution of leptospirosis cases according to severity compared in children and adults.

The outcome of treated patients was as follows: $87.62 \%$ of cases survived and were discharged after mean hospital treatment $14.9 \pm 7.3$ days (from 1 to 46 days) and $12.38 \%$ was with lethal outcome after mean hospital treatment $4.2 \pm 2.6$ days (from 1 to 10 days). All of deceased patients were in the group of adults (OR 32.4; $\mathrm{p}<0.0005$ ).

The clinical onset of leptospirosis in deceased patients was meanly five days before admission in hospital. All of them had fever, muscular pains, oligo/anuria, two had epistaxis and hemorrhagic rash before admission. Ten deceased patients had co-morbidity including hypertonic disease and chronic alcohol abuse (respectively three cases), past myocardial infarction, stomach ulcer, past tuberculosis (respectively two), podagra and calculous cholecystitis (respectively one).

All patients with unfavorable outcome had, besides ARF, at least two other major organ failures. Other abnormalities were affected consciousness, multi-site hemorrhagic diathesis (in nine). The major factors leading to death were lung edema and brain edema (OR 25.00; $\varphi=0.659082$ and 17.29; $\varphi=0.527778$, respectively) due to severe ARF.

Pathomorphological investigations were performed in seven deceased cases. Macroscopically, severe lung edema, brain edema leading to cerebellar inclination, multisite bleeding, enlarged congestive liver were established in all of autopsied, pancreatitis in five, peritonitis in one. The histological investigations had demonstrated gastrointestinal and myocardial hemorrhages, focal myocardial necrosis, destruction of liver architectonic, severe tubular necrosis of kidneys in all investigated.

\section{Discussion}

Leptospirosis is a zoonosis of global importance caused by Leptospira interrogans complex. The disease is seasonal, with peak incidence occurring in summer or fall in temperate regions, where temperature is the limiting factor in survival of leptospires, and during rainy seasons in warm-climate regions, where rapid desiccation would otherwise prevent survival [1]. Bulgaria is a country with temperate climate and our study confirms mentioned above observations. Summer seasonal predominance was observed $(79 \%)$. There was not difference in seasonal distribution of cases in groups of children and adults.

Leptospirosis is a direct zoonosis. Leptospires are maintained in nature by a large variety of animal hosts. Leptospires shed in the urine of carrier animals can survive in the environment for prolonged period. The source of human leptospiral infection is infected animal urine. Contact with various species of animals, animal tissue, animal urine and wet environment and occupational and recreational exposure to contaminated water bodies have been implicated as risk factors $[15,16]$. In our study, 93 (89\%) of all cases had risk exposures to contaminated water, rodents or domestic animals. All of children and $87 \%$ of adults had risk exposures to one or more than one risk factors. Precise obtained information about premorbid risk exposures plays a crucial role for early clinical diagnosis of leptospirosis.

The demographic characteristics of the cases in our study match those described in the literature, albeit with an increased proportion of older patients. The predominance of male cases is generally attributed to the hypothesis that men are potentially more exposed than women due to more frequent at-risk activities. However, Cassadou et al (2016) observed this ratio in the older age group where women are more numerous than men and probably have similar daily activities, suggesting that other hypothesis needs to be evaluated [17]. In our study, only ten female cases was registered $(9.52 \%)$ and male are prevalent both in children and adults groups.

Regarding the clinical characteristic, fever (100\%), headache, (92\%), hepatomegaly $(85 \%)$, nausea and vomiting (85\%), myalgia (77\%) and shivering (77\%) were the commonest symptoms in children and $31 \%$ of children cases were icteric. These observations correlate with other studies of pediatric leptospirosis [18]. The prevalence of splenomegaly, jaundice, oliguria, abdominal pain, diarrhea and arrhythmia was significantly higher in adults. In the study of Rajajee et al (2002) among 139 children with leptospirosis, the commonest symptoms were fever (96\%), headache and myalgia (24\%). Jaundice was present in only $18 \%$ of cases with renal failure. Hepatomegaly was observed in $72 \%, 9 \%$ of children presented with shock and $7 \%$ had meningitis [19]. Beside of this we consider that it should be kept in mind the observation of Silva HR et al (2003) that leptospiral infection in children is underestimated, because it is asymptomatic or presents as a cold syndrome simile [20].

Leptospirosis is re-emerging zoonosis with broad clinical spectrum from anicteric and usually self-limiting acute febrile illness to icteric and occasionally fatal form. Icteric leptospirosis is a much more severe disease in which the clinical course is often very rapidly progressive. Between 5 and $10 \%$ of leptospirosis cases have icteric form. The jaundice in leptospirosis is not associated with hepatocellular necrosis, and liver function returns to normal after recovery. Serum bilirubin levels may be high, and many weeks may be 
required for normalization. There are moderate rises in transaminases levels [1]. It was well demonstrated in our study, that mild to moderately elevated transaminases levels were seen both in whole series and different age groups contrasting to high serum bilirubin levels. Totally $64 \%$ of cases were icteric $31 \%$ of cases from group of children and $68 \%$ of adults group; $\mathrm{p}<0.005)$. Transaminases levels in adults were significantly higher than those in children $(\mathrm{p}<0.0005)$.

Other important feature of leptospirosis is renal involvement. It is varied from transient oliguria to ARF, which occurs in 16 to $40 \%$ of cases. In patients with ARF, oliguria (OR 9.98) is a significant predictor of death [quoted in 1]. We consider that when ARF is presented, leptospirosis is a serious infection with severe multi-systemic organ involvement and ultimately failure. In the absence of ARF, death due to leptospirosis is very uncommon, but the reported mortality due to leptospirosis with ARF is high: $36 \%$ in Barbados [1], 26\% in Sri Lanka [8], and 17\% in Turkey [21]. In our study $31 \%$ of children cases had oliguria vs. $62 \%$ of adults $(\mathrm{p}<0.025)$ but only $15 \%$ of children had ARF vs. $67 \%$ of adults $(p<0.0005)$. All thirteen deceased patients $(12 \%)$ had severe ARF, leading to lung edema and brain edema, which were causes for the death. This observations match to those of Daher EF et al (2014) which considered that ARF was more frequent in adults and it was associated with increased mortality [22]. In study of Spichler A et al (2012) was mentioned that compared with children, adults had higher rates of jaundice, elevated serum bilirubin levels, oliguria, and elevated creatinine levels but not for thrombocytopenia or pulmonary involvement. The overall case-fatality rate was $27 \%$ (adult leptospirosis) versus $5 \%$ (pediatric) $(\mathrm{P}<0.01)$. Severe pediatric leptospirosis may be less likely to show all classic features of Weil's disease and may be less fatal than in adults [23] but according to Guerrier $\mathrm{G}$ et al (2013) severe leptospirosis in adolescents may be more likely to show adults' characteristics compared with children [24]. Lopes AA et al (2003) had concluded that pediatric patients have a lower risk of death during hospitalization than adults with leptospirosis [25].

Pulmonary symptoms in cases of leptospirosis may be the major manifestations of leptospirosis in some clusters of cases and in some sporadic cases. The severity of respiratory disease is unrelated to the presence of jaundice. Patients may present with a spectrum of symptoms, ranging from cough, dyspnea, and hemoptysis to ARDS. Intraalveolar hemorrhage was detected in the majority of patients, even in the absence of overt pulmonary symptoms. Pulmonary hemorrhage may be severe enough to cause death [1]. During the past decade, there has been a global increase in recognition of the severe pulmonary form of leptospirosis and Weil's disease with pulmonary involvement. In the study of Spichler A et al (2008) pulmonary involvement was the strongest prognostic factor (OR, 6.0) [10]. In our study we did not observe severe pulmonary form of leptospirosis without ARF. Acute respiratory failure had observed only in adults (15\%) as a part of multi-organ failure $(\mathrm{p}<0.0005)$.

Cardiac involvement in leptospirosis is common but may be underestimated. Evidence of myocardial involvement was detected in $10 \%$ of 80 severe icteric cases in Louisiana [1], while in India and Sri Lanka - in over $40 \%$ of patients [7, 8, 26-28]. Arrhythmias were predictors of myocarditis (OR 5.774) in severe leptospirosis cases in Sri Lanka [8], and in Brazilian series (OR 2.83) [9]. Repolarization abnormalities on ECG were considered a poor prognostic indicator (OR 5.9) in severe leptospirosis cases. A mortality rate of 54\% was reported in severe leptospirosis with myocarditis [29]. The presence of myocarditis was strongly associated with lethal outcome and this consideration was confirmed in study of Shah K et al (2010) by pathomorphological investigation of twenty-four hearts from patients died from leptospirosis. Myocarditis was noted in $96 \%$ of cases [26]. We had no found ECG abnormalities suspected for myocarditis and cardiac arrhythmias in group of children while in adults these findings were $23 \%$ and $15 \%$, respectively $(p<0.0005)$.

Thrombocytopenia occurs in $\geq 50 \%$ of leptospirosis cases and is a significant predictor for development of ARF. However, thrombocytopenia in leptospirosis is transient and does not result from disseminated intravascular coagulation (DIC) [1]. Low platelet counts are common in leptospirosis, and human and experimental data have been inconsistent in supporting a role for an underlying DIC in predisposing to hemorrhagic manifestations in leptospirosis [30]. One potential explanation of thrombocytopenia in leptospirosis is that certain strains of Leptospira directly activate platelets [10]. We had established thrombocytopenia in $59 \%$ of cases despite hemorrhagic diathesis had been observed in $37 \%$. That discrepancy could be explained with prompt intensive and supportive treatment.

Aseptic meningitis may be found in $\leq 25 \%$ of all leptospirosis cases and may account for a significant minority of all causes of aseptic meningitis. Patients with aseptic meningitis have tended to be younger than those with icteric leptospirosis. In their series of 616 cases, Alston and Broom noted that $62 \%$ of children $\leq 14$ years old presented with aseptic meningitis [quoted to 1]. In our study aseptic meningitis was found in $31 \%$ of children and $18 \%$ of adults.

Serum amylase levels are often raised significantly in association with ARF, but clinically apparent pancreatitis is not common. Necrotizing pancreatitis has been detected on autopsy [1]. We had observed clinically apparent pancreatitis only in seven adult cases (four of them with lethal outcome). One of autopsied patients had necrotizing pancreatitis complicated to sero-fibrinous peritonitis.

\section{Conclusion}

Leptospirosis in Pleven region is not common but had presented severe course in 32\%. Leptospirosis in children usually is mild and anicteric. The leptospiral meningitis is not unusual manifestation for pediatric leptospirosis. The major factors leading to death in adults with leptospirosis were lung edema and brain edema due to severe ARF. 


\section{References}

[1] Levett PN: Leptospirosis. Clin Microbiol Rev, 2001; 14: 296326.

[2] Lozano R, Naghavi M, Foreman K, Lim S, Shibuya K, Aboyans V. et al. Global and regional mortality from 235 causes of death for 20 age groups in 1990 and 2010: a systematic analysis for the Global Burden of Disease Study 2010. Lancet, 2012; 380: 2095-128

[3] Costa F, Hagan JE, Calcagno J, Kane M, Torgerson P, Martinez-Silveira MS, et al. Global morbidity and mortality of leptospirosis: A systematic review. PLoS Negl Trop Dis, 2015; 9:e0003898.

[4] Torgerson PR, Hagan JE, Costa F, Calcagno J, Kane M, Martinez-Silveira MS et al. Global burden of leptospirosis: estimated in terms of disability adjusted life years. Plos Negl Trop Dis, 2015; 9:e0004122

[5] Panaphut T, Domrongkitchaiporn S, Thinkamrop B. Prognostic factors of death in leptospirosis: a prospective cohort study in Khon Kaen, Thailand. Int J Infect Dis, 2002; 6: $52-59$.

[6] Tantitanawat S, Tanjatham S. Prognostic factors associated with severe leptospirosis. J Med Assoc Thai, 2003; 86: 925-931.

[7] Pappachan MJ, Mathew S, Aravindan KP, Khader A, Bharghavan PV, Kareem MM, et al. Risk factors for mortality in patients with leptospirosis during an epidemic in northern Kerala. Natl Med J India, 2004; 17: 240-242.

[8] Dassanayake D, Wimalaratna H, Nandadewa D, Nugaliyadda A, Ratnatunga C, Agampodi S. Predictors of the development of myocarditis or acute renal failure in patients with leptospirosis: An observational study. BMC Infectious Diseases, 2012; 12: 4.

[9] Ko AI, Galvao Reis M, Ribeiro Dourado CM, Johnson WD Jr, Riley LW. Urban epidemic of severe leptospirosis in Brazil. Lancet, 1999; 354: 820-825.

[10] Spichler A, Vilaça P, Athanazio D, Albuquerque J, Buzzar M, Castro B, et al. Predictors of lethality in severe leptospirosis in urban Brazil. Am J Trop Med Hyg, 2008; 79: 911-914.

[11] Gancheva G. Complex studies on leptospirosis. PhD dissertation, Medical University - Pleven, Bulgaria, 2006: 7273. Bulgarian.

[12] Tasseva E, Christova I, Gladnishka T, Trifonova I, Ivanova V. Leptospirosis in humans in Bulgaria - a new challenge for medicine or problem solved. Retrospective analysis of infection for 2010-2011. Science Infectology/ Parasitology, 2012; 1: 22-29. Bulgarian.

[13] Costa E, Costa YA, Lopes AA, Sacramento E, Bina JC. Severe forms of leptospirosis: clinical, demographic and environmental aspects. Rev Soc Bras Med Trop, 2001; 34: 261-267.

[14] DEPARTMENT OF INTERNATIONAL ECONOMIC AND SOCIAL AFFAIRS. PROVISIONAL GUIDELINES ON STANDARD INTERNATIONAL AGE CLASSIFICATIONS. UNITED NATIONS New York, 1982; Series M No. 74: p. 8.

[15] Vijayashari P, Sugunan AP and Shiram AN. Leptospirosis: an emerging global public health problem. $J$ Biosci, 2008; 33 (4): 557-569.

[16] Mori M, Van Esbroeck M, Depoorter S, Decaluwe W, Vandecasteele SJ, Fretin D, et al. Outbreak of leptospirosis during a scout camp in the Luxembourg Belgian province, Belgium, summer 2012. Epidemiol Infect. 2015; 143 (8): 1761-6.

[17] Cassadou S, Rosine J, Flamand C, Escher M, Ledrans M, Bourhy P, et al. Underestimation of Leptospirosis Incidence in the French West Indies. PLOS Negl Trop Dis, April 29, 2016. | DOI: 10. 1371/journal. pntd. 0004668.

[18] Suárez Hernández M, Martínez Sánchez R, Posada Fernández P, Bustelo Aguila J, Carrera Nodal O, Bravo Fleite F, et al. Leptospirosis in children in Ciego de Avila Province, Cuba. Rev Soc Brasil Med Trop, 1999; 32 (2): 145-150.

[19] Rajajee S, Shankar J and Dhattatri L. Pediatric presentations of leptospirosis. Indian J Pediatr, 2002; 69: 851.

[20] Silva HR, Tavares-Neto J, Bina JC and Meyer R. Leptospiral infection and subclinical presentation among children in Salvador, Bahia. Rev Soc Brasil Med Trop, 2003; 36 (2): 227-233.

[21] Turhan V, Polat E, Murat Atasoyu E, Ozmen N, Kucukardali Y, Cavuslu S. Leptospirosis in Istanbul, Turkey: a wide spectrum in clinical course and complications. Scand J Infect Dis, 2006; 38: 845-852.

[22] Daher EF, Vieira AP, Jacinto $\mathrm{CN}$, Lima RS, Girão MM, Fernandes AT, et al. Differences among children, adolescents and adults with severe leptospirosis: A comparative analysis. Indian J Nephrol. 2014; 24 (3): 166-70.

[23] Spichler A, Athanazio DA, Vilaça P, Seguro A, Vinetz J, Leake JA. Comparative analysis of severe pediatric and adult leptospirosis in Sao Paulo, Brazil. Am J Trop Med Hyg. 2012; 86 (2): 306-308.

[24] Guerrier G, Hie P, Gourinat AC, Huguon E, Polfrit Y, Goarant $\mathrm{C}$ et al. Association between age and severity to leptospirosis in children. PLoS Negl Trop Dis. 2013; 7 (9): e 2436.

[25] Lopes AA, Costa E, Costa YA, Sacramento E, Oliveira Jr ARR, Lopes MB et al. Comparative study of the in-hospital case-fatality rate of leptospirosis between pediatric and adult patients of different age groups. Rev. Inst. Med. trop. S. Paulo, 2004; 46 (1): 19-24.

[26] Shah K, Amonkar GP, Kamat RN, Deshpande JR. Cardiac findings in leptospirosis. J Clin Pathol, 2010; 2: 119-123.

[27] Karande S, Kulkarni H, Kulkarni K, De A and Varaiya V. Leptospirosis in Children in Mumbai Slums. Ind J Pediatr, 2002; 69: 855-858.

[28] Narayanan R, Sumathi G, Prabhakaran SG, Shanmughapriya S, Natarajaseenivasan K. Paediatric leptospirosis: A population based case-control study from Chennai, India. Indian J Med Microbiol. 2016; 34 (2): 228-32.

[29] Dupont H, Dupont-Perdrizet D, Perie JL, Zehner-Hansen S, Jarrige B, and Daijardin JB. Leptospirosis: prognostic factors associated with mortality. Clin Infect Dis, 1997; 25: 720-724.

[30] Wagenaar JF, Goris MG, Sakundarno MS, Gasem MH, Mairuhu AT, de Kruif MD, et al. What role do coagulation disorders play in the pathogenesis of leptospirosis? Trop Med Int Health, 2007; 12: 111-122. 